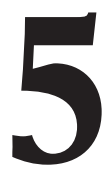

\title{
¿QUIÉNES DEBEN SER LOS AGENTES EVALUADORES DEL TFG?
}

\section{(WHO SHOULD BE THE ASSESSMENT AGENTS OF THE FINAL YEAR PROJECT?)}

Carmen Isabel Reyes García

Alicia Díaz Megolla

Universidad de Las Palmas de Gran Canaria

DOI: $10.5944 / e d u c X X 1.23843$

\section{Cómo referenciar este artículo/How to reference this article:}

Reyes García, C.I. y Díaz Megolla, A. (2020). ¿Quiénes deben ser los agentes evaluadores del TFG? Educación XX1, 23(1), 125-145, doi: 10.5944/educXX1.23843

Reyes García, C.I. \& Díaz Megolla, A. (2020). Who should be the assessment agents of the final year project? Educación XX1, 23(1), 125-145, doi: 10.5944/educXX1.23843

\section{RESUMEN}

Uno de los principios fundamentales del enfoque de evaluación alternativo demandado desde el Espacio Europeo de Educación Superior (EEES) es desarrollar una evaluación participativa. Evaluar el aprendizaje desde este enfoque, de manera integral y formativa, implica recoger la información desde las diferentes perspectivas de los/las agentes que intervienen en la evaluación. La responsabilidad de la evaluación es un tema que cobra una especial importancia en la asignatura del Trabajo Fin de Grado (TFG) dadas las características particulares de esta asignatura. Por ello, nuestro trabajo se centra en ofrecer una propuesta de evaluación participativa y analizar los agentes evaluadores que establece la normativa de las universidades públicas españolas para comprobar si esta potencia una evaluación participativa y democrática como se reclama desde el EEES. 
Para ello se realizó un estudio descriptivo mediante el análisis de documentos y se emplearon como unidades de análisis las normativas que regulan la evaluación del TFG en las universidades públicas españolas. Los resultados de este estudio indican que el marco normativo de la evaluación del TFG no fomenta una evaluación participativa, ya que, en la mayor parte de las universidades la responsabilidad recae en el tribunal. Mientras que el alumnado como agente evaluador es ignorado y la persona que ejerce la tutoría desempeña únicamente una función formativa y solo, en unas pocas universidades desarrolla una función sumativa de la evaluación. Por este motivo, las universidades españolas tienen un reto de cara al futuro: modificar la normativa del TFG y orientarla hacia la promoción de una evaluación participativa.

\section{PALABRAS CLAVE}

Educación Superior; trabajo fin de grado; autoevaluación; evaluación formativa; evaluación sumativa; evaluación del aprendizaje.

\section{ABSTRACT}

One of the fundamental principles of the alternative assessment approach demanded by the European Higher Education Area (EHEA) is the participation of the agents involved in the assessment. Assessing learning from this approach, in an integral and formative way, involves collecting information from the different perspectives of the agents involved in the assessment process. The assessment agents are an issue that takes on special importance in the subject of Final Year Project (FYP) given its contextual characteristics: the difficulty involved in assessing a subject consisting mainly of a large number of transversal competences, the wide range of different formats that can be used to assess, and the diversity of agents involved in the assessment of the FYP (board, teacher-tutor as well as other professionals from companies or external institutions, and students). Therefore, our work focuses on analyzing the assessment agents established by the regulations of the Spanish public universities to verify, if this enhances a participatory and democratic assessment as claimed from the EHEA.

A descriptive study was conducted through the analysis of documents and regulations governing the FYP in Spanish public universities used as units of analysis. The results of this study indicate that the normative framework of the assessment of the FYP does not encourage a participatory assessment since, in most universities, the responsibility lies on the boards. While the student as an assessment agent is ignored and the tutor plays 
only a formative role and only a few universities develop a summative role of the assessment. For this reason, Spanish universities have a challenge for the future: modify the regulations of the FYP and guide it towards the promotion of a participatory assessment.

\section{KEY WORDS}

Higher Education; Final year project; self-assessment; formativeassessment; summative-assessment; learning assessment.

\section{INTRODUCCIÓN}

Uno de los aspectos más polémicos en las universidades españolas tras el proceso de Bolonia ha sido la aparición de los Trabajos Fin de Grado (TFG) en los planes de estudio. Después de varios años de implantación, esta asignatura sigue generando una gran discusión entre teóricos y prácticos de la Educación Superior. La evaluación constituye uno de los problemas centrales de esta materia dadas sus características contextuales: la dificultad que supone evaluar una materia constituida por competencias trasversales, la amplitud de formatos a través de los que puede ser evaluada y la diversidad de agentes que pueden participar en la evaluación del TFG (tribunales, profesorado-tutor de distintos departamentos, muchas veces sin experiencia en este tipo de trabajo, así como otros profesionales de empresas o instituciones externas, y el alumnado).

El objetivo de este trabajo es, por una parte, ofrecer una propuesta participativa sobre quienes deben ser las personas responsables en la evaluación del TFG y por otra, analizar el reglamento de evaluación de los TFG de las universidades públicas españolas para determinar si este promueve uno de los principios básicos del enfoque de evaluación actual: la evaluación participativa.

\section{EVALUACIÓN DEL TFG}

En los últimos años se han publicado muchos estudios en torno a la evaluación del TFG, tanto a nivel nacional como internacional. Los países anglosajones tienen una mayor tradición respecto a este tipo de trabajos denominados, entre otros términos, como Dissertation y Final Year Project (Healey, Jenkins \& Lea, 2014). En nuestro país, también se han realizado estudios relacionados con diversos aspectos de la evaluación 
de esta asignatura: el proceso de evaluación (Freire et al, 2015); la mejora del proceso de valoración de los resultados (Díaz-Vázquez, GarcíaDíaz, Maside \& Vázquez-Rozas, 2018); y sobre evaluación y tutorización (Vera \& Briones, 2015). En el contexto iberoamericano las publicaciones consultadas sobre la evaluación del TFG, denominado tesis de pregrado, se centran fundamentalmente en las dificultades que tiene el estudiantado para terminar su trabajo de licenciatura debido a la falta de tutorización continuada y la falta de formación metodológica (Sáez, 2005).

Los TFG surgen a raíz del Real Decreto (RD) 1393/2007, por el que se establece la ordenación de las enseñanzas universitarias oficiales, y su actualización en el RD 861/2010, señala que todas las enseñanzas oficiales concluirán con la elaboración y defensa de un Trabajo Fin de Título (TFT). A partir de este momento, el TFG se convierte en una materia obligatoria que se desarrollará al final del plan de estudios y que estará orientada a la evaluación de competencias asociadas al título.

En nuestro país, la evaluación de competencias surge en los años 90 como consecuencia de una de las demandas del Espacio Europeo de Educación Superior (EEES). Este nuevo planteamiento evaluativo implicó la aparición de un modelo que rompió con la evaluación tradicional afirmando que el objetivo principal de la evaluación es promocionar el aprendizaje del estudiantado (Bilbao \& Villa, 2019, Bordas \& Cabrera, 2001, Fraile, Pardo \& Panadero, 2018; Gil \& Padilla, 2009; Ibarra \& Rodríguez, 2010; Mateo \& Vlachopoulos, 2019). Este modelo alternativo ha recibido diferentes denominaciones: evaluación formativa, evaluación de competencias, evaluación auténtica, evaluación orientada al aprendizaje, etc. Todas ellas comparten el objetivo anterior, aunque sostienen sus propias matizaciones. Una evidencia de la presencia de este modelo en nuestras universidades lo constituye la Red de Evaluación Formativa y Compartida en la Docencia Universitaria que surge en el año 2005. Esta Red ya cuenta con más de 30 universidades y su finalidad es la mejora del aprendizaje universitario en el EEES, a través de propuestas de evaluación formativa y compartida (Hamodi \& López, 2015).

Desde este modelo, evaluar un TFG implica defender una evaluación formativa y continua integrada en el proceso de enseñanza-aprendizaje (Boud \& Associates, 2010); alineada con los objetivos (Biggs, 2005); desarrollada mediante el desempeño de tareas complejas (Brockbank \& McGill, 2002) que reflejen o simulen al máximo las situaciones de la vida real (Gulikers, Bastiaens \& Kirschner, 2006); que impliquen el feedback del aprendizaje (Carless, 2007; Gibbs, 2010); que aporten criterios y estándares claros, utilizando instrumentos de evaluación adecuados (Ibarra \& Rodríguez, 2010; Mateo \& Vlachopoulos, 2010) y que implique procesos participativos 
y democráticos donde los estudiantes intervengan en la evaluación (Álvarez, Padilla, Rodríguez, Torres \& Suárez, 2011; Boud \& Falchikov, 2006; Tejada \& Ruiz, 2016). En este último presupuesto, los agentes evaluadores, se centrará nuestro trabajo.

\section{AGENTES EVALUADORES DEL TFG}

Uno de los principios básicos del modelo actual de evaluación es que la evaluación no debe recaer solo en el profesorado, sino que debe ser compartida con otros agentes implicados (Bordas \& Cabrera, 2001). Este es uno de los cambios que supuso el nuevo paradigma de evaluación defendido por el EEES. Tal y como manifiesta Ríos y Herrera (2017) es imposible evaluar, de manera integral y formativa, las competencias de los/las estudiantes a partir de un único agente evaluador. Es necesario recoger la información desde la perspectiva de cada una de las personas que intervienen en las acciones y procedimientos que realiza el estudiantado para evidenciar sus procesos y logros de aprendizaje. Tejada y Ruiz (2016) defienden que este nuevo enfoque reclama procesos más participativos y democráticos que incluyan procedimientos para llegar a acuerdos que integren las diferentes perspectivas valoradas.

La responsabilidad de la evaluación cobra una especial importancia en la asignatura del TFG dado el contexto particular en que se desarrolla. El número y la naturaleza de las competencias a evaluar en el TFG, exige la participación de diferentes agentes que valoren las distintas competencias asociadas al título a través de diferentes acciones y procedimientos en diferentes escenarios y momentos.

En los últimos años, ha habido cierta producción de trabajos centrados en estudiar y determinar los/las responsables de la evaluación del TFG, aunque hoy por hoy, no existe un acuerdo unánime en sus propuestas. Diferentes autores han investigado como agentes evaluadores al tribunal y al tutor/a, estando la calificación o función sumativa relegada al tribunal (Bonilla, Fuentes, Vacas \& Vacas, 2012; Freire et al. 2015; Valderrama et al. 2010).

Por otra parte, otros estudios, siguiendo más de cerca la perspectiva del EEES, defienden la implicación del alumnado en la evaluación, incluyendo algún tipo de modalidad de participación del estudiantado en la valoración del TFG (Hernández-Leo et al. 2013; Mateo, Escofet, Martínez \& Ventura, 2009; Rekalde-Rodríguez; Rodríguez e Ruiz de Gauna \& Bilbao, 2018; Rodríguez e Ibarra, 2014; Rullan, Fernández, Estapé \& Márquez, 2010). 
Nuestra propuesta se ubica en esta segunda línea de trabajo, ya que defendemos una evaluación participativa del TFG donde las personas responsables de la evaluación valoren no solo el resultado final del trabajo (función sumativa), sino también el proceso que ha seguido el alumnado en la elaboración (función formativa). A continuación, presentamos el conjunto de agentes que deberían tener alguna responsabilidad en la evaluación y esbozamos las principales funciones que deberían desempeñar (Figura 1).

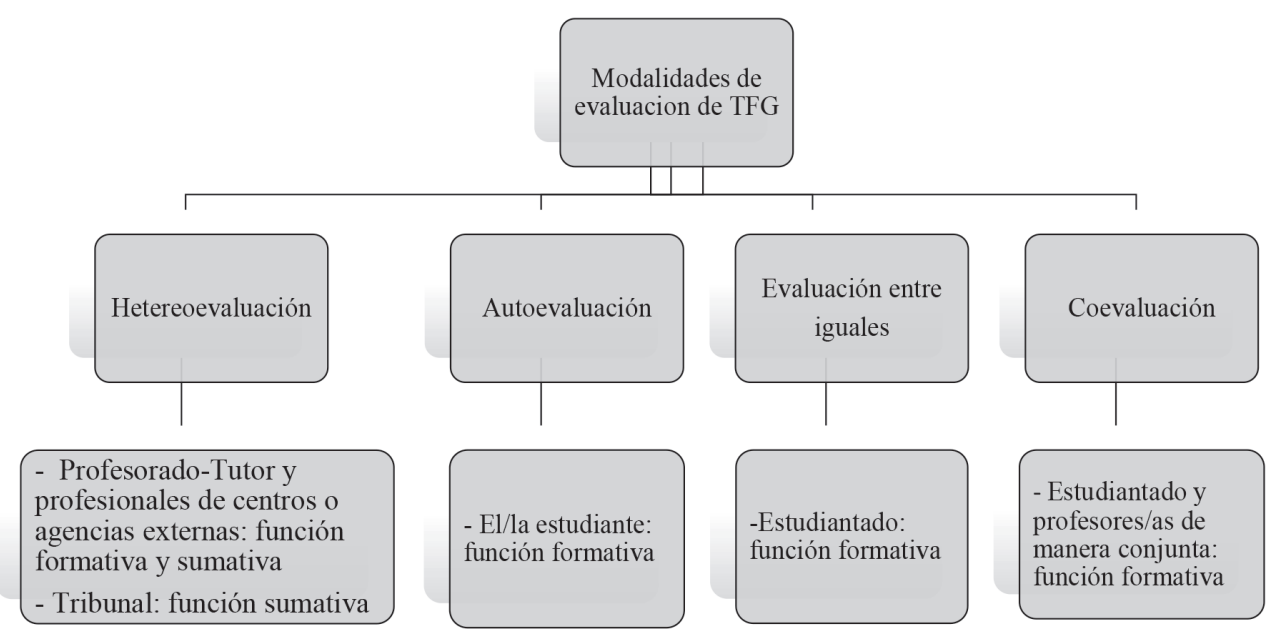

Figura 1. Modalidades y funciones de la evaluación del TFG atendiendo a los agentes

En primer lugar, señalaremos al profesorado-tutor cuya función principal es orientar y supervisar durante la realización del TFG. Consideramos que esta función implica la evaluación formativa, ya que el/la tutor/a deberá ir aportando feedback a los/las estudiantes sobre la calidad de sus realizaciones durante la elaboración del TFG con el objetivo de promover y mejorar el aprendizaje. Por otra parte, los/las tutores/as también deberían tener la responsabilidad de asignar una parte de la calificación final del TFG. Solo así se podrán calificar competencias y actitudes tan importantes como la capacidad del estudiantado en la gestión y planificación del tiempo, la autonomía, el esfuerzo, la iniciativa o la comunicación interpersonal. Por tanto, el profesorado-tutor del TFG debe desarrollar tanto la función formativa como la función sumativa (Rekalde-Rodríguez et al. 2018). Dentro de esta categoría de profesorado-tutor incluimos a profesionales de instituciones o empresas externas, dado que el TFG puede también desarrollarse en empresas o instituciones externas donde un miembro de la misma podrá actuar de cotutor/a.

En segundo lugar, aludiremos a los tribunales. Los TFG deberán ser evaluados por una comisión o tribunal, es decir, por un grupo de profesores/ 
as, que calificarán el resultado final del trabajo, así como la presentación y defensa del mismo. Aunque en la práctica, probablemente debido al elevado número de estudiantes, la presentación y defensa no siempre tiene lugar, consideramos que esta debe desarrollarse en todos los casos, ya que tal y como exponen Mateo et al. (2009) "La función de esta comisión es principalmente juzgar la capacidad comunicativa del alumnado y el modo en el que es capaz de presentar información científica ante un público cualificado" (p.65). Por lo tanto, el tribunal garantiza la objetividad de la evaluación final del TFG desarrollando así una función sumativa. De esta forma, a pesar de que, tanto el/la tutor/a como tribunal, desarrollarán una función sumativa o calificadora, lo harán sobre actuaciones desarrolladas en momentos diferentes por el alumnado y desde diferentes perspectivas.

Por último, nombraremos al estudiantado. Desde el enfoque actual de evaluación, los/las estudiantes deben estar implicados/as como agentes evaluadores, ya que, con ello se favorece el desarrollo de su aprendizaje a lo largo de toda la vida. El alumnado debe tener responsabilidad como evaluador de su aprendizaje pues esto: "implica una toma de conciencia sobre las propias posibilidades y limitaciones, asumir los déficits de aprendizaje y consecuentemente, desarrollar una actitud positiva para superarlos" (Gil \& Padilla, 2009: 47). Por lo tanto, la participación del estudiantado es una de las condiciones necesarias para que la evaluación promueva el aprendizaje. Esto se podrá realizar a través de diferentes modalidades: la evaluación entre iguales (los/as estudiantes valoran los trabajos o actuaciones de sus compañeros/as); la coevaluación (el/la estudiante valora su trabajo o actuación de manera negociada con el profesorado); o la autoevaluación (la valoración de su propio trabajo) (Álvarez et al. 2011; Rullan et al. 2010; To \& Panadero, 2019). Sin embargo, queremos puntualizar que, la autoevaluación o self-assessment no implica necesariamente la autocalificación o selfgrading, ya que, poner una nota, no supone realizar un análisis profundo de un trabajo (Fraile, Pardo \& Panadero, 2018). Defendemos la modalidad de la autoevaluación entendiéndola como la valoración cualitativa que el/ la estudiante realiza a lo largo y al final del proceso de aprendizaje sobre su propio trabajo analizando sus fortalezas y debilidades con la intención de mejorarlo. Consideramos, junto a Fraile et al. (2018), que la parte más importante de la autoevaluación es la reflexión del estudiante sobre su propio trabajo, ya que esto favorecerá su capacidad de autorregulación del aprendizaje. Sin embargo, a pesar de las dificultades que supone, no desestimamos que el/la estudiante pueda calificarse al final su trabajo asignándose una nota. Por otra parte, pensamos que la autoevaluación procesual precisa de un diálogo constante con el/la tutor/a por lo que estimamos que la autoevaluación implica la coevaluación, es decir, la evaluación negociada entre tutor/a y estudiante donde la responsabilidad es 
compartida. Esta modalidad permite al estudiantado ser más consciente de los errores y aprender de ellos (Quesada, Gómez, Gallego \& Cubero, 2019).

Por lo tanto, proponemos que la evaluación del TFG atendiendo a los agentes evaluadores, se debe desarrollar a través de diferentes modalidades: la heteroevaluación (la responsabilidad de evaluar recae en agentes externos: el tribunal y el profesorado-tutor e incluso, en algunos casos, profesionales de instituciones o empresas externas) y la autoevaluación, coevaluación y evaluación entre iguales. En cualquiera de estas tres modalidades (autoevaluación, coevaluación y evaluación entre iguales) la participación de los/las estudiantes en la evaluación, debería tener, al menos, una función formativa aportando una valoración cualitativa de su trabajo.

Por otra parte, la participación conjunta de diferentes agentes evaluadores no supone que todos deban tener el mismo peso en la calificación ya que se tendrá que ponderar el papel de cada uno/a (Tejada \& Ruiz, 2016).

Contrariamente a todo lo expuesto, la responsabilidad compartida de la evaluación prácticamente no existe en las aulas de nuestras universidades donde continúa desarrollándose a través de agentes externos (heteroevaluación) (Álvarez et al, 2011; Bilbao \& Villa, 2019).

Uno de los factores que puede estar contribuyendo a que la evaluación participativa no se desarrolle en la práctica, puede provenir de la normativa reguladora del TFG. Por ello, el objetivo de este trabajo es ofrecer una propuesta de evaluación participativa y analizar los responsables de la evaluación del TFG que establece la normativa de las universidades públicas españolas, así como las funciones de la evaluación que desempeñan para comprobar, si esta potencia una evaluación participativa y democrática como se demanda desde el EEES.

\section{MÉTODO}

En el presente estudio realizamos una investigación cualitativa (Rodríguez, Gil \& García, 1996), a través de la técnica de análisis de documentos. Se estableció un sistema categorial inductivo, de manera que la entrada conceptual de cada categoría se definió una vez que se había revisado toda la información. El sistema categorial quedó configurado por una dimensión denominada evaluación participativa, la cual defiende que los agentes evaluadores deben ser todos aquellos que están implicados en la evaluación. 
Una vez establecidas las dos categorías de agentes evaluadores (Tribunal y Tutor/a), analizamos las diferentes subcategorías que podíamos encontrar por parte de estos agentes. Nos interesaba conocer qué función/es evaluadora/s (formativa o sumativa) se les asignaba en la normativa, ya que ambas son necesarias en el proceso de evaluación. Así, observamos que, en el caso del Tribunal, se le atribuía una función sumativa, donde su papel era calificar el trabajo y defensa del TFG. Se podían dar dos situaciones: 1) El tribunal puede incluir al tutor/a del TFG; 2) El tribunal no puede incluir al tutor/a del TFG. En aquellos casos donde no se decía explícitamente que el/la tutor/a podía formar parte del tribunal, entendíamos que no podía incluirse.

En cuanto al Tutor/a quedó patente su función formativa, ya que en la normativa se citaban sus responsabilidades, como la orientación, seguimiento, apoyo, etc. De ahí, asumimos que la función formativa por parte del tutor/a se cumple en todas las universidades estudiadas y, por ello, consideramos innecesario representarlo en las tablas de resultados. Respecto a su función sumativa, nos encontramos con las siguientes situaciones: 1) El/la tutor/a tiene un peso en la calificación final del trabajo, que puede ir desde el 100\% (evalúa solo), hasta un porcentaje determinado (por ejemplo, 30\%) o indeterminado (se menciona que debe ponderarse su calificación, pero no cuánto); 2) Por otra parte, también se halló que el/a tutor/a realiza un informe, con mayor o menor grado de detalle, sobre la realización del trabajo y este debe ser considerado por parte del tribunal para la calificación aunque también puede no ser considerado; 3 ) Al tutor/a se le da audiencia pudiendo tener voz (pero no voto) con anterioridad a la calificación del tribunal. Se introdujeron estas dos últimas subcategorías (informe y audiencia) porque son aspectos o situaciones que, de alguna forma, están aludiendo a la función evaluadora y nuestra intención era poder dilucidar, si el/la tutor/a ejercía la función sumativa en esos casos.

A pesar de que una de las condiciones necesarias para que la evaluación favorezca el aprendizaje, implica incluir al estudiantado, evidenciamos cómo ninguna universidad hace partícipe al alumnado en la evaluación del TFG. En la Tabla 1 se presenta cómo quedó configurado el sistema categorial atendiendo a la función sumativa. 
Tabla 1

Sistema categorial del análisis de la normativa de los agentes evaluadores del TFG

\begin{tabular}{l|c|c|c|c|c|c|c}
\hline \multicolumn{7}{c}{ Dimensión evaluación participativa } \\
\hline Categorías & $\begin{array}{c}\text { Agente } \\
\text { evaluador: } \\
\text { Tribunal }\end{array}$ & \multicolumn{5}{|c}{$\begin{array}{c}\text { Agente evaluador: } \\
\text { Tutor/a }\end{array}$} \\
\hline \multirow{3}{*}{ Subcategorías } & $\begin{array}{c}\text { Con } \\
\text { tutor/a }\end{array}$ & $\begin{array}{c}\text { Sin } \\
\text { tutor/a }\end{array}$ & $\begin{array}{c}\text { Ponderación de la } \\
\text { calificación }\end{array}$ & \multicolumn{2}{|c|}{ Informe } & Audiencia \\
\cline { 2 - 8 } & & $100 \%$ & $\begin{array}{c}\text { Se } \\
\text { señala } \\
\text { un \% } \%\end{array}$ & $\begin{array}{c}\text { No se } \\
\text { señala } \\
\%\end{array}$ & $\begin{array}{c}\text { Se } \\
\text { considera }\end{array}$ & $\begin{array}{c}\text { No se } \\
\text { considera }\end{array}$ & Con voz \\
\hline
\end{tabular}

Para realizar el análisis documental, se recogió de manera sistemática la normativa de evaluación del segundo nivel de concreción curricular, es decir, aquella normativa propia de cada una de las universidades públicas españolas donde se determinaba quién debe realizar la evaluación del TFG. La finalidad de esta muestra documental fue conocer quién/es son las personas responsables de evaluar el TFG y qué funciones cumplen, según el marco regulador que sustenta nuestras universidades para, a partir de aquí, comprobar, si esta potencia una evaluación participativa y democrática como se reclama desde el EEES.

\section{MUESTRA}

La muestra de documentos normativos fue identificada y seleccionada a través de las páginas web institucionales de cada una de las 48 universidades públicas españolas. En el análisis de documentos no incluimos las normativas de las universidades de Córdoba; Autónoma de Barcelona; Barcelona; Lleida; Politécnica de Cataluña; Rovira i Virgili y Politécnica de Madrid, que en el momento de la última revisión (enero 2019) no disponían de normativa específica para el TFG, o tenían una normativa muy genérica donde únicamente se instaba a los centros a establecer sus propias normas. Por tanto, la muestra final quedó constituída por 41 documentos.

\section{MATERIALES Y PROCEDIMIENTO}

En cuanto a la búsqueda de la información, las normativas se encontraron en las páginas web de las distintas universidades. Una vez obtenidos los documentos normativos, aplicamos el sistema de categorías 
mencionado anteriormente (Tabla 1). Con el objetivo de organizar los diferentes datos obtenidos de estos análisis, se construyó una tabla de doble entrada donde se volcó toda esa información (Tabla 2). La Tabla 2 recoge en las filas, las diferentes universidades (ordenadas alfabéticamente por comunidad autónoma) y en las columnas, los agentes que participan en la evaluación del TFG: Tribunal y Tutor. En las columnas se incluyeron igualmente las subcategorías relacionadas con las situaciones que deben cumplir los agentes en referencia a la evaluación. Finalmente, señalábamos con una " $\mathrm{X}$ " los agentes y situaciones que aparecían en la normativa revisada de cada universidad.

Tabla 2

Agentes de evaluación del TFG y situaciones que cumplen según la normativa universitaria

\begin{tabular}{|c|c|c|c|c|c|c|c|c|}
\hline & \multirow{2}{*}{\multicolumn{2}{|c|}{$\begin{array}{c}\text { Tribunal } \\
\text { Califica }\end{array}$}} & \multicolumn{6}{|c|}{ Tutor } \\
\hline & & & \multicolumn{3}{|c|}{$\begin{array}{l}\text { Ponderación de la } \\
\text { calificación }\end{array}$} & \multicolumn{2}{|c|}{ Informe } & \multirow{2}{*}{$\begin{array}{c}\text { Audiencia } \\
\text { Con voz }\end{array}$} \\
\hline & $\begin{array}{c}\text { Con } \\
\text { Tutor/a }\end{array}$ & $\begin{array}{c}\text { Sin } \\
\text { Tutor/a }\end{array}$ & $100 \%$ & $\begin{array}{c}\text { Señala } \\
\text { un } \%\end{array}$ & $\begin{array}{c}\text { No } \\
\text { señala } \\
\text { un \% } \\
\end{array}$ & $\begin{array}{c}\mathrm{Se} \\
\text { considera }\end{array}$ & $\begin{array}{c}\text { No se } \\
\text { considera }\end{array}$ & \\
\hline 1.Almería & & $\mathrm{X}$ & & & $\mathrm{X}$ & $\mathrm{X}$ & & \\
\hline 2. Cádiz & & $\mathrm{X}$ & & & & & $\mathrm{X}$ & \\
\hline 3.Granada & & $\mathrm{X}$ & & & & $\mathrm{X}$ & & \\
\hline 4.Huelva & $\mathrm{X}$ & & & & & & $\mathrm{X}$ & $\mathrm{X}$ \\
\hline 5.Jaén & $\mathrm{X}$ & & & & & & $\mathrm{X}$ & $\mathrm{X}$ \\
\hline 6.Málaga & & $\mathrm{X}$ & & & & $\mathrm{X}$ & & \\
\hline 7.Pablo de Olavide & & $\mathrm{X}$ & & & & & $\mathrm{X}$ & \\
\hline 8.Sevilla & & $\mathrm{X}$ & & & & & $\mathrm{X}$ & $\mathrm{X}$ \\
\hline 9.Zaragoza & & $\mathrm{X}$ & & & & & $\mathrm{X}$ & \\
\hline 10.Oviedo & & $\mathrm{X}$ & & & & $\mathrm{X}$ & & \\
\hline 11.Islas Baleares & & $\mathrm{X}$ & $\mathrm{X}$ & & & & $\mathrm{X}$ & \\
\hline 12.La Laguna & & $\mathrm{X}$ & $\mathrm{X}$ & $\mathrm{X}$ & & & $\mathrm{X}$ & \\
\hline 13.Las Palmas de GC & & $\mathrm{X}$ & & & & & $\mathrm{X}$ & \\
\hline 14.Cantabria & & $\mathrm{X}$ & $\mathrm{X}$ & & & & $\mathrm{X}$ & \\
\hline $\begin{array}{l}\text { 15.Castilla- } \\
\text { LaMancha }\end{array}$ & $\mathrm{X}$ & & & & & $\mathrm{X}$ & & \\
\hline 16. Burgos & & $\mathrm{X}$ & & & & & & \\
\hline 17.León & & $\mathrm{X}$ & & & & $\mathrm{X}$ & & \\
\hline 18.Salamanca & & $\mathrm{X}$ & & & & $\mathrm{X}$ & & \\
\hline
\end{tabular}




\begin{tabular}{|c|c|c|c|c|c|c|c|c|}
\hline & \multirow{2}{*}{\multicolumn{2}{|c|}{$\begin{array}{c}\text { Tribunal } \\
\text { Califica }\end{array}$}} & \multicolumn{6}{|c|}{ Tutor } \\
\hline & & & \multicolumn{3}{|c|}{$\begin{array}{l}\text { Ponderación de la } \\
\text { calificación }\end{array}$} & \multicolumn{2}{|c|}{ Informe } & \multirow{2}{*}{$\begin{array}{c}\text { Audiencia } \\
\text { Con voz }\end{array}$} \\
\hline & $\begin{array}{c}\text { Con } \\
\text { Tutor/a }\end{array}$ & $\begin{array}{c}\text { Sin } \\
\text { Tutor/a }\end{array}$ & $100 \%$ & $\begin{array}{c}\text { Señala } \\
\text { un } \%\end{array}$ & $\begin{array}{c}\text { No } \\
\text { señala } \\
\text { un \% } \\
\end{array}$ & $\begin{array}{c}\mathrm{Se} \\
\text { considera }\end{array}$ & $\begin{array}{c}\text { No se } \\
\text { considera }\end{array}$ & \\
\hline 19.Valladolid & & $\mathrm{X}$ & & & & & $\mathrm{X}$ & $\mathrm{X}$ \\
\hline 20.Girona & $\mathrm{X}$ & & & & & $\mathrm{X}$ & & \\
\hline 21.Jaume I & & $\mathrm{X}$ & $\mathrm{X}$ & & & $\mathrm{X}$ & & \\
\hline 22.Pompeu Fabra & $\mathrm{X}$ & & & & & & $\mathrm{X}$ & \\
\hline 23.Extremadura & & $\mathrm{X}$ & & & & & $\mathrm{X}$ & \\
\hline 24.A Coruña & & $\mathrm{X}$ & & & & & & \\
\hline $\begin{array}{l}\text { 25.Santiago } \\
\text { Compostela }\end{array}$ & $\mathrm{X}$ & & & & & $\mathrm{X}$ & & $\mathrm{X}$ \\
\hline 26.Vigo & & $\mathrm{X}$ & & $\mathrm{X}$ & & $\mathrm{X}$ & & \\
\hline 27.La Rioja & & $\mathrm{X}$ & & & & & $\mathrm{X}$ & \\
\hline 28.Alcalá & $\mathrm{X}$ & & $\mathrm{X}$ & & & $\mathrm{X}$ & & \\
\hline $\begin{array}{l}\text { 29.Autónoma de } \\
\text { Madrid }\end{array}$ & $\mathrm{X}$ & & $\mathrm{X}$ & & & & & \\
\hline 30.Carlos III & $\mathrm{X}$ & & & & & & $\mathrm{X}$ & \\
\hline $\begin{array}{l}\text { 31.Complutense de } \\
\text { Madrid }\end{array}$ & $\mathrm{X}$ & & & & & & $\mathrm{X}$ & \\
\hline 32.Rey Juan Carlos & & $\mathrm{X}$ & & & & & $\mathrm{X}$ & \\
\hline 33.UNED & $\mathrm{X}$ & & $\mathrm{X}$ & & & $\mathrm{X}$ & & \\
\hline 34.Murcia & & $\mathrm{X}$ & & & $\mathrm{X}$ & $\mathrm{X}$ & & \\
\hline $\begin{array}{l}\text { 35. Politécnica de } \\
\text { Cartagena }\end{array}$ & $\mathrm{X}$ & & & & & & $\mathrm{X}$ & \\
\hline $\begin{array}{l}\text { 36. Pública de } \\
\text { Navarra }\end{array}$ & & $\mathrm{X}$ & & & & & $\mathrm{X}$ & \\
\hline 37.País Vasco & & $\mathrm{X}$ & & & & $\mathrm{X}$ & & \\
\hline 38.Alicante & & $\mathrm{X}$ & & & & & & \\
\hline $\begin{array}{l}\text { 39. Miguel } \\
\text { Hernández }\end{array}$ & & $\mathrm{X}$ & & & $\mathrm{X}$ & $\mathrm{X}$ & & \\
\hline $\begin{array}{l}\text { 40. Politécnica de } \\
\text { Valencia }\end{array}$ & $\mathrm{X}$ & & & & & & $\mathrm{X}$ & $\mathrm{X}$ \\
\hline 41. Valencia & & $\mathrm{X}$ & $\mathrm{X}$ & & & & & \\
\hline
\end{tabular}

Con el objetivo de garantizar una adecuada fiabilidad en el análisis de los documentos, este procedimiento se llevó a cabo en dos ocasiones por parte de cada investigadora de manera independiente y posteriormente 
fue consensuado entre ambas, obteniendo un total grado de acuerdo en los análisis independientes. Una vez hecho esto los datos obtenidos fueron volcados en una base de datos donde se realizó el análisis de frecuencia y los porcentajes.

\section{RESULTADOS}

Los resultados que se presentan en la Tabla 3 recogen la información referente al análisis de la normativa de evaluación del TFG de las 41 universidades públicas españolas, que se presentan en la Tabla 2. En esta observamos cómo todas las universidades coinciden en que la evaluación del TFG debe realizarse por parte de un tribunal.

Tabla 3

Participación de los Agentes evaluadores del TFG en la normativa de las universidades españolas

\begin{tabular}{|c|c|c|c|c|c|c|}
\hline $\begin{array}{c}\text { Agente } \\
\text { evaluador: } \\
\text { Tribunal }\end{array}$ & \multicolumn{6}{|c|}{$\begin{array}{c}\text { Agente evaluador: } \\
\text { Tutor/a }\end{array}$} \\
\hline \multirow[t]{2}{*}{$\begin{array}{l}\text { Con } \\
\text { tutor }\end{array}$} & \multicolumn{3}{|c|}{$\begin{array}{l}\text { Ponderación de la } \\
\text { calificación }\end{array}$} & \multicolumn{2}{|c|}{ Informe } & \multirow{2}{*}{$\begin{array}{c}\text { Audiencia } \\
\text { Con voz }\end{array}$} \\
\hline & $100 \%$ & $\begin{array}{l}\text { Señala } \\
\text { un } \%\end{array}$ & $\begin{array}{c}\text { No señala } \\
\text { un } \%\end{array}$ & $\begin{array}{c}\text { Se } \\
\text { considera }\end{array}$ & $\begin{array}{c}\text { No se } \\
\text { considera }\end{array}$ & \\
\hline $31,7 \% \quad 68,3 \%$ & $19,5 \%$ & $4,8 \%$ & $7,3 \%$ & $39 \%$ & $48,9 \%$ & $14,6 \%$ \\
\hline
\end{tabular}

En relación al Tribunal, el 68,3\% de las normativas señalan que la persona que tutoriza el trabajo no debe formar parte del mismo o en caso de hacerlo debe abstenerse ("El Director del Trabajo Fin de Grado, en el caso de ser miembro de la Comisión de Evaluación, se abstendrá de la misma en el momento de presentar su tutelado el Trabajo dirigido"). Por otro lado, en el 31,7\% de los documentos se indica que el/a tutor/a puede formar parte del tribunal ("A criterio de la Comisión de TFG de la Facultad o Escuela, la persona encargada de la tutoría del TFG podrá ser miembro del Tribunal que lo evalúe"). En referencia a este resultado consideramos que, dado que el tribunal debe cumplir una función sumativa y final de la evaluación, la participación del tutor/a puede condicionar este tipo de evaluación, debido a que el conocimiento que tiene sobre el proceso de realización del TFG puede interferir en su valoración como miembro del tribunal. Por esta razón, entendemos que no debe formar parte del tribunal, ya que la función del tribunal es evaluar de forma objetiva el resultado final del trabajo y la 
defensa del mismo, y la participación del tutor/a puede restar objetividad a esta labor.

En cuanto a la función sumativa o de calificación que puede desarrollar el/la Tutor/a, observamos cómo en un 19,5\% de los casos es quien se encarga de calificar al 100\% el TFG ("los directores de los trabajos sean los encargados del proceso de defensa y evaluación de los mismos"). En otros casos, concretamente en un 4,8\%, se explicita la ponderación mínima que debe tener el/la tutor/a en la calificación ("la evaluación del tutor o tutora, con una ponderación mínima del $20 \%$ sobre la calificación final"). Por último, en un 7,3\% de los casos, se señala que la calificación del tutor/a debe estar ponderada, pero no se indica en qué porcentaje ("la Comisión Evaluadora tendrá en cuenta la ponderación que tenga establecida entre la calificación del director y la calificación de la propia Comisión Evaluadora"). En cuanto a estos resultados, vemos cómo en algunos casos el/la tutor/a califica al $100 \%$, esta situación se plantea como una posibilidad que se propone a los centros, probablemente justificada por el número elevado de trabajos presentados. En otros casos, observamos que esto ocurre en relación con la oferta de dos modalidades de trabajo: general y específica. En la denominada modalidad general, el tutor se encarga de un grupo de estudiantes, como en cualquier asignatura, por lo que será el único agente de evaluación del trabajo. Consideramos que, independientemente de la circunstancia, el hecho de que el/la tutor/a evalúe al 100\% el TFG implica que se reduzca a un solo agente la evaluación, privando al alumnado del carácter objetivo que implica la evaluación externa de un tribunal. En este sentido, entendemos que la situación idónea es aquella en las que se indica que el/la tutor/a debe tener una cierta ponderación en la calificación, ya que implica que asignará una parte de la nota y el tribunal adjudicará el resto de la calificación. Esta situación se presenta únicamente en la normativa de cinco universidades (Almería, La Laguna, Vigo, Murcia y Miguel Hernández) lo que constituye un $12,2 \%$ de los casos.

En referencia al Informe que realiza el/la tutor/a sobre el TFG, vemos cómo el 39\% de las normativas señalan que debe ser considerado por parte del tribunal, frente al $48,9 \%$ en el que no se señala de forma explícita que este documento deba ser considerado por parte del tribunal, aunque se indique que debe realizarse. En este sentido, en relación a la consideración del informe por parte del tribunal nos encontramos situaciones excepcionales, como en la universidad Rey Juan Carlos I, donde por un lado se plantea que el/la tutor/a debe realizar un informe para autorizar la presentación del trabajo, pero a su vez el alumnado puede presentarlo sin su consentimiento. Situaciones como estas u otras, donde se insta al tutor/a a realizar un informe, pero no se especifica en qué medida este debe ser considerado por el tribunal, conlleva a que no quede claro hasta qué punto 
el informe es vinculante o qué peso debe tener para el tribunal. Por ello, dada la información tan ambigua, no podemos dilucidar si la normativa de estas universidades atribuye una función sumativa al tutor/a a través del informe. Por último, en relación a la Audiencia que se le da al tutor/a antes de la deliberación por parte del tribunal, observamos cómo un 14,6\% de las normativas explicitan que el/la tutor/a podrá ser oído antes de que el tribunal califique. En todos los casos se trata de una participación sin voto, donde se ofrece esta posibilidad en cualquier circunstancia ("el tutor podrá solicitar audiencia"; "el tutor será oído, si así lo desea") o únicamente en caso de suspenso ("la comisión evaluadora dará audiencia al tutor antes de otorgar "suspenso"). En cualquier caso, no se especifica el peso que tendrá esta intervención, ya que queda a juicio del tribunal el impacto que tiene esta información en la calificación final. Por esta razón, entendemos que, al ser esta medida tan poco precisa, el/la tutor/a sigue sin tener función sumativa.

\section{DISCUSIÓN}

Según los documentos normativos, en la mayor parte de nuestras universidades se atribuye al tribunal la función sumativa o calificadora de la evaluación del TFG. Este resultado se opone a nuestra propuesta, que defiende una evaluación participativa donde los diferentes agentes evaluadores actuarán, de manera complementaria, en la evaluación del TFG. Tampoco somos partidarias, como ocurre en algunas universidades, de que el/la tutor/a pueda pertenecer al tribunal, ya que la participación del tutor/a en la valoración de la presentación y defensa del tribunal, puede condicionar la evaluación debido a que el conocimiento que tiene sobre el proceso de realización del TFG, puede mermar la objetividad de la evaluación externa. Su evaluación debe encaminarse, por un lado, a la evaluación formativa, es decir a valorar aspectos procesuales relacionados con el desarrollo de competencias a lo largo del proceso de elaboración del TFG (i.e. analizar si el/la estudiante incorpora de manera autónoma información adicional para apoyar y completar los contenidos; organiza y desarrolla, de manera autónoma, un plan de trabajo ajustado). Por otro lado, su evaluación también debe ser sumativa, pero no del resultado final del trabajo y de la presentación y defensa del TFG (que es cometido del tribunal) sino calificar las competencias desarrolladas por los/las estudiantes a lo largo del proceso de elaboración del TFG.

Otros hallazgos importantes hacen referencia a la función formativa que la normativa asigna al tutor/a, ya que, lo define como: "La persona encargada del seguimiento, apoyo, orientación, etc. del TFG”. Por lo que la persona que ejerce la tutoría proveerá información a lo largo del proceso 
sobre la calidad del trabajo. Sin embargo, a diferencia de nuestra propuesta, los documentos no reconocen la función sumativa del tutor/a salvo en unas pocas universidades donde le atribuyen parte de la calificación. Dado que el/ la tutor/a es el único agente que podrá calificar competencias relacionadas con el proceso de elaboración del trabajo, nos resulta paradójico que la normativa no le atribuya parte de la calificación y la nota esté supeditada generalmente a la calificación final del tribunal. Los/a profesores/as que hemos sido tutores/as del TFG, sabemos que el resultado final de un trabajo no aporta información sobre el desarrollo del mismo, de manera que podemos encontrar dos trabajos similares en cuanto a la calidad que, sin embargo, merezcan una calificación diferente. Esta diferencia en la calificación puede deberse al tiempo de dedicación, tutorías y ayuda que ha requerido un/a estudiante frente a otro/a que ha sido mucho más autónomo/a y que ha necesitado menos correcciones y ayuda. Consideramos, junto a Tejada y Ruiz (2016), que se necesitan las perspectivas de diferentes agentes en la evaluación de competencias en Educación Superior. En el caso del TFG, se deben tener en cuenta, por un lado, la perspectiva del tutor/a que estará referida al proceso de desarrollo de competencias y, por otro, la del tribunal que se relacionará con la presentación y defensa del trabajo. Se trata de escenarios y momentos distintos; en el primer caso el/la tutor/a evaluará progresivamente el proceso de elaboración a través de las tutorías presenciales, virtuales, telefónicas, exposiciones y producciones de los estudiantes en diferentes contextos: despacho, tutoría virtual, teléfono, sala de presentaciones, biblioteca,... Mientras que el tribunal, evaluará al final del proceso en base al trabajo final y al momento de exposición y defensa en la sala asignada para ello. Por tanto, es obvio pensar que la calificación final del TFG deba ser asignada por ambos agentes. Por la misma razón, tampoco defendemos el planteamiento de algunas universidades que en su normativa proclaman al tutor/a como único agente evaluador, ya que solo mediante la complementariedad de la información y perspectivas, es posible desarrollar la evaluación integral del estudiantado. Por tanto, ni el tribunal, ni el/la tutor/a deben ser agentes únicos de la evaluación del TFG, ya que un único agente evaluador no puede abarcar la diversidad de competencias que desarrollan los/las estudiantes en las diferentes fases del TFG.

En cuanto a la implicación del estudiantado en la evaluación, resulta sorprendente que no haya ninguna universidad que introduzca en la normativa alguna modalidad de participación del alumnado. Esto nos hace pensar que el marco normativo de la evaluación del TFG de las universidades españolas está al margen de la abundante investigación que ha demostrado los beneficios de la misma y que afirma que es imprescindible implicar al alumnado en la evaluación, si realmente se quiere promocionar el aprendizaje (Álvarez et al, 2011; Boud \& Falchikov, 2006; Carless, 2007; Fraile et al., 2018, Hamodi \& López, 2015). Por tanto, este resultado tampoco 
está en consonancia con nuestra propuesta que aboga por la implicación de los/las estudiantes desarrollando una evaluación formativa a través de la autoevaluación y la coevaluación, de manera que los/las estudiantes valoren de manera cualitativa su propio trabajo a lo largo de todo el proceso de elaboración emitiendo juicios y consensuándolos con el/la tutor/a a través del diálogo constante.

En síntesis, el tribunal es el único agente evaluador de los TFG en la mayor parte de las universidades españolas. El/la tutor/a actúa fundamentalmente como evaluador formativo $\mathrm{y}$, solamente en unas pocas universidades se le permite calificar conjuntamente con el tribunal los TFG. El estudiantado no tiene ninguna responsabilidad en la evaluación del TFG. Lo que nos hace pensar, junto a Fook y Sidhu (2014), que cada vez es mayor la distancia entre los conocimientos y las habilidades que el alumnado adquiere y aquellos otros que son necesarios para tener "éxito" en el lugar de trabajo del siglo XXI. En definitiva, la hereoevaluación es la modalidad de evaluación utilizada y esta se desarrolla en casi todos los casos a través de un único agente evaluador (el tribunal). Por tanto, el marco normativo de las universidades españolas no propone una evaluación participativa. En un reciente estudio, Bilbao y Villa (2019) hallaron que entre todos los presupuestos que defiende el EEES, la evaluación participativa es el que menos se está llevando a cabo. Por todo ello, consideramos que se hace necesario introducir un planteamiento más democrático en la normativa de evaluación del TFG y acorde a las demandas del EEES, de manera que incluya a los agentes evaluadores distinguiendo sus funciones: el tribunal con una función sumativa, el/la tutor/a con una función formativa y sumativa y al estudiantado al menos con una función formativa a través de la autoevaluación y la coevaluación. Somos conscientes de que implementar estos cambios no es una tarea fácil debido a la cultura instalada en las universidades, la resistencia del profesorado y la falta de formación de profesores y estudiantes, entre otros aspectos. Por ello, pensamos que el cambio se debe hacer procesualmente. Así, por ejemplo, en el caso de la participación del estudiantado en la evaluación, el marco normativo de las universidades podría empezar por incluir explícitamente la realización de un autoinforme (autoevaluación) como una parte o elemento del TFG, así como la realización de tutorías a lo largo del proceso de elaboración del TFG donde el profesorado-tutor y el alumnado confronten valoraciones sobre la calidad del trabajo desarrollado (coevaluación). Estas dos medidas ayudarían a promocionar que los/las estudiantes sean capaces de autorregular su propio proceso de aprendizaje. 


\section{REFERENCIAS BIBLIOGRÁFICAS}

Álvarez, V., Padilla, M.T., Rodríguez, J., Torres, J. \& Suárez, M. (2011). Análisis de la participación del alumnado universitario en la evaluación de su aprendizaje. Revista Española de Pedagogía, 250, 401-426.

Biggs, J. (2005). Calidad del aprendizaje universitario. Madrid: Narcea.

Bilbao, A. \& Villa, A. (2019). Avances y limitaciones en la evaluación del aprendizaje a partir del proceso de convergencia. Visión docente y discente en los grados de Educación Infantil y Primaria. Educación XX1, 22(1), 45-69, doi: 10.5944/ educXX1.19976.

Bonilla, M ${ }^{\mathrm{a} J ., ~ F u e n t e s, ~ L ., ~ V a c a s, ~ C . ~ \& ~}$ Vacas, T. (2012) Análisis del proceso de evaluación del Trabajo Fin de Grado en las nuevas titulaciones. Educade Revista de Educación en Contabilidad, Finanzas y Administración de Empresas, 3, 5-21.

Bordas, M. I. \& Cabrera, F. A. (2001). Estrategias de evaluación de los aprendizajes centrados en el proceso. Revista Española de Pedagogía, 218, 25-48.

Boud, D. \& Associates 2020. (2010). Seven propositions for assessment reform in higher education. Australian Learning and Teaching Council.

Boud, D. \& Falchikov, N. (2006). Aligning assessment with long-term learning. Assessment \& Evaluation in Higher Education, 31(4), 399-413. doi: $10.1080 / 02602930600679050$.

Brockbank, A. \& McGill, I. (2002). Aprendizaje reflexivo en Educación Superior. Madrid: Morata.

Carless, D. (2007). Learning-oriented assessment: conceptual bases and practical implications. Innovations in Education and Teaching International, 44(1), 57-66. doi: 10.1080/14703290601081332.

Díaz-Vázquez, R., García-Díaz, A., Maside, J.M. \& Vázquez-Rozas, E. (2018). El Trabajo de Fin de Grado: fines, modalidades y estilos de tutorización. REDU. Revista de Docencia Universitaria. 16(2), juliodiciembre, 159-175. doi: 10.4995/ redu.2018.10178.

Fook, C. \& Sidhu, G. (2014). Assessment practices in higher education in United States. Procedia-Social and Behavioral Sciences, 123, 299-306. doi:10.1016/j. sbspro.2014.01.1427.

Fraile, J., Pardo, A. \& Panadero, E. (2018). Autoevaluación y autocalificación en el grado en ciencias de la actividad física y del deporte: estudio censal de las guías docentes. Profesorado. Revista de Currículum y Formación, 22(3), (Julio- Septiembre, 163-182. doi: 10.30827/profesorado.v22i3.7997.

Freire, P., Díaz, R., Martínez, F., Maside, J., Del Rio, Mª́ \&. Vázquez, E. (2015). Valoración del proceso de enseñanzaaprendizaje en el Trabajo Fin de Grado. REDU: Revista de docencia universitaria, 13(2), 323-344. doi 10.4995/redu.2015.5451. Recuperado de http://bit.ly/2WZSbgh

Gibbs. G (2010). Using assessment to support student learning. Reino Unido: Leeds Metropolitan University. Recuperado de http://bit.ly/2LR8SJs

Gil, J. \& Padilla, $M^{\mathrm{a}}$ T. (2009). La participación del alumnado universitario en la evaluación del aprendizaje. Educación, XX1, 12, 4365. 
Gulikers, J., Bastiaens, Th. \& Kirschner, P. (2006). Relations between student perception assessment authenticity, study approaches and learning outcomes. Studies in Educational Evaluation, (32), 381-400. doi:10.1016/j.stueduc.2006.10.003.

Hamodi, C. \& López, V. (2015). Medios, técnicas e instrumentos de evaluación formativa y compartida del aprendizaje en educación superior. Perfiles Educativos, XXXVII (147), 146161.

Healey, M., Jenkins, A., \& Lea, J. (2014). Developing research-based curricula in college-based higher education. New York: Higher Education Academy. Recuperado de http://bit.ly/2w9Z7eF

Hernández-Leo, D., Moreno, V., Camps, I., Clarisó, R., Martínez-Monés, A., Marco-Galindo, MJ. \& Melero, J. (2013). Implementación de buenas prácticas en los Trabajos Fin de Grado. Revista de Docencia Universitaria, 11, 259-278.

Ibarra, S. y Rodríguez, G. (2010). Los procedimientos de evaluación como elementos de desarrollo de la función orientadora en la universidad. Revista Española de Orientación y Psicopedagogía, 21(2), 443-461.

Ibarra, $M^{\mathrm{a}}$.S. \& Rodríguez, G. (2014). Modalidades participativas de evaluación: Un análisis de la percepción del profesorado y de los estudiantes universitarios. Revista de Investigación Educativa, 32(2), 339361. http://bit.ly/2YFMzrP

Mateo, J., Escofet, A., Martínez, F. \& Ventura, J. (2009). Guía para la evaluación de competencias en el trabajo de fin de grado en el ámbito de las ciencias sociales y jurídicas. Barcelona: Generalitat de Catalunya.
Agència per a la Qualitat del Sistema Universitaria Catalunya.

Mateo, J. \& Vlachopoulos, D. (2010). La nueva naturaleza del aprendizaje y de la evaluación en el contexto del desarrollo competencial, retos europeos en la educación del SXXI. Revista Iberoamericana de Evaluación, 3(3), 44-61. Recuperado de http://bit. ly/2HtajK5

Quesada, V., Gómez, M., Gallego, $M^{a}$.B. \& Cubero-Ibáñez, J. (2019). Should I use co-assessment in higher education? Pros and cons from teachers and students' perspectives. Assessment \& Evaluation in Higher Education, 44(7), 987-1002. doi: 10.1080/02602938.2018.1531970.

Real Decreto 1393/2007, de 29 de octubre, por el que se establece la ordenación de las enseñanzas universitarias oficiales (BOE 30-1007).

Real Decreto 861/2010, de 2 de julio, por el que se modifica el Real Decreto 1393/2007, de 29 de octubre, por el que se establece la ordenación de las enseñanzas universitarias oficiales (BOE-03-07-10).

Rekalde-Rodríguez, I., Ruiz de Gauna, M. \& Bilbao, B. (2018) Acción tutorial y evaluación formativa en los Trabajos de Fin de Grados. REDU. Revista de Docencia Universitaria. 16(2), juliodiciembre, 123-141. doi: 10.4995/ redu.2018.10185.

Ríos, D. \& Herrera, D. (2017). Los desafíos de la evaluación por competencias en el ámbito educativo. Educ. Pesqui. 43(4), 1073-1086. http:// bit.ly/2w7Wie9

Rodríguez, G., Gil, J. \& García, E. (1996). Metodología de la investigación cualitativa. Málaga: Aljibe. 
Rullan, M., Fernández, M., Estapé, G. \& Márquez, M. (2010). La evaluación de competencias transversales en la materia Trabajos Fin de Gado. Un estudio preliminar sobre la necesidad y oportunidad de establecer medios e instrumentos por ramas de conocimiento. Revista de Docencia Universitaria, 8(1),74-100.

Tejada, J. \& Ruiz, C. (2016). Evaluación de competencias profesionales en Educación Superior: Retos e implicaciones. Educación XX1, 19(1), 17-38, doi:10.5944/educXX1.12175.

To, J. \& Panadero, E. (2019). Peer assessment effects on the self-assessment process of first-year undergraduates, Assessment \& Evaluation in Higher Education, 44(6), 920-932. doi: 10.1080/02602938.2018.1548559. Recuperado de http://bit.ly/2w6RaXV
Sáez, V. (2005). La investigación en pregrado: revisión de aspectos metodológicos en la elaboración del trabajo de licenciatura, Escuela de Geografía, Universidad Central de Venezuela, Venezuela. Terra Nueva Etapa, XXI (30), 97-113.

Valderrama, E., Rullán, M., Sánchez, F., Pons, J., Mans, C., Giné, F., Seco, G., Jiménez, L., Peig, E., Carrera, J., Moreno, A., García, J., Pérez, J., Vilanova, R., Cores, F., Renau. J.M., Tejero, J. \& Bisbal, J. (2010). La evaluación de competencias en los Trabajos Fin de Estudios. IEE-RITA 5(3), 107-114.

Vera, J. \& Briones, E. (2015). Perspectiva del alumnado de los procesos de tutorización y evaluación de los trabajos de fin de grado. Cultura $y$ Educación, 27(4),742-765. doi: 10.1080/11356405.2015.1089391. 


\section{PERFIL ACADÉMICO Y PROFESIONAL DE LAS AUTORAS}

Carmen Isabel Reyes García. Licenciada en Psicología y Pedagogía, doctora en Psicopedagogía y profesora del Departamento de Educación de la Universidad de Las Palmas de Gran Canaria (ULPGC). Coordina el Grupo de Innovación educativa de la ULPGC: Evaluación del Aprendizaje Universitario. Ha colaborado en la formación didáctica del profesorado en los diferentes niveles educativos, especialmente, en educación superior. Su línea de investigación es la evaluación del aprendizaje universitario, donde ha publicado libros y artículos en revistas científicas nacionales e internacionales.

Alicia Díaz Megolla. Doctora en Psicología y premio extraordinario de doctorado por la Universidad de La Laguna (ULL). Profesora del Departamento de Educación de la Universidad de Las Palmas de Gran Canaria (ULPGC) desde 2011. Es miembro del grupo de investigación "Dificultades de Aprendizaje, Psiconlingüística y Tecnologías de la Información y de la Comunicación" de la ULL, del grupo de investigación "Educación Inclusiva" de la ULPGC y del grupo de innovación "Evaluación del aprendizaje universitario" de la ULPGC.

Dirección de las autoras: C/Santa Juana de Arco, $\mathrm{n}^{\circ} 1$ 35004 - Las Palmas de Gran Canaria

E-mail: carmen.reyes@ulpgc.es alicia.diaz@ulpgc.es

Fecha Recepción del Artículo: 14. Febrero. 2019

Fecha Modificación del Artículo: 12. Mayo. 2019

Fecha Aceptación del Artículo: 20. Mayo. 2019

Fecha Revisión para Publicación: 08. julio. 2019 
